\title{
Construction of multiple shRNA vectors targeting PEDV and TGEV and production of transgenic SCNT porcine embryos in vitro
}

\author{
Jianwen CHEN $^{1 *}$, Kaiyuan PAN ${ }^{1 *}$, Zhen CHEN $^{1}$, Biao DING ${ }^{1}$, Dandan SONG ${ }^{1}$, Wenbin BAO (凶) $)^{2}$, \\ Yunhai ZHANG (ه) $)^{1}$
}

1 Anhui Provincial Laboratory of Local Livestock and Poultry, Genetical Resource Conservation and Breeding, College of Animal Science and Technology, Anhui Agricultural University, Hefei 230036, China

2 College of Animal Science and Technology, Yangzhou University, Yangzhou 225009, China

\begin{abstract}
Porcine viral diarrhea is an acute and highly contagious enteric disease of pigs that causes huge economic losses worldwide. Porcine epidemic diarrhea virus (PEDV) and transmissible gastroenteritis virus (TGEV) are the main pathogens responsible for piglet viral diarrhea. However, currently there is no specific drug available for the effective treatment of viral diarrhea. Therefore, it is necessary to seek an effective method to diminish PEDV and TGEV infection rates. RNA interference has been applied successfully to inhibit the virus replication. It provides a potential strategy for breeding resistant pigs. In this study, four promoters and four short hairpin RNA (shRNA) vectors with LoxP sites at each end of the selectable marker genes were constructed to target PEDV and TGEV. These vectors were then transfected into porcine fetal fibroblasts, G418 resistant transfectants were confirmed by PCR and transgenic SCNT porcine blastocysts were obtained. These results have paved the way for future production of marker-free transgenic resistant to PEDV and TEGV pigs by SCNT.
\end{abstract}

Keywords piglet diarrhea, RNAi, PEDV, TGEV, transgenic resistance breeding

\section{Introduction}

Porcine epidemic diarrhea virus (PEDV) is an acute and highly contagious enteric disease characterized by severe enteritis, vomiting and watery diarrhea, and constitutes a

Received December 24, 2017; accepted February 9, 2018

Correspondences: wbbao1974@163.com, yunhaizhang@ahau.edu.cn

${ }^{*}$ These authors contribute equally to the work major problem for the swine industry. PEDV was first reported in Belgium, UK in $1971^{[1]}$ and PEDV infections are a continuing problem worldwide in the swine industry. Since December 2010, large-scale outbreaks of diarrhea with $80 \%-100 \%$ morbidity and $50 \%-90 \%$ mortality in suckling piglets have occurred in most Chinese provinces, affecting even vaccinated pigs ${ }^{[2,3]}$. Investigations indicated that these large-scale outbreaks of diarrhea were caused by a variant of PEDV ${ }^{[4,5]}$. In 2013, new PEDV outbreaks also occurred in Republic of Korea, Thailand, Vietnam and the USA.

Transmissible gastroenteritis virus (TGEV) is also identified as one of the most important pathogenic agents in swine. TGEV causes acute enteritis in swine of all ages, especially in neonatal piglets, for which the mortality rate is close to $100 \%{ }^{[6,7]}$, leading to severe annual economic loss in swine-producing areas. Despite years of intensive vaccine research, TGEV infections remain a severe problem in the swine industry worldwide, resulting in huge economic losses and public health concerns. Hence, it is crucial to breed a new pig lines that are resistant to PEDV and TGEV.

RNA interference (RNAi) is a powerful new method for intracellular immunization against viral infection. The introduction of small interfering RNA (siRNA) into mammalian cells can activate RNAi, resulting in sequence-specific degradation of the invasive RNA. It has been applied successfully to inhibit the replication of animal viruses ${ }^{[8-17]}$, providing a potential strategy for farm animal breeding applications. Recent advances involving the use of RNAi-based technologies present alternative approaches for inhibition of PEDV and TGEV replication in vitro ${ }^{[18-20]}$. However, one problem is that siRNA cannot entirely inhibit virus replication. It has been suggested that siRNA activity loss of effectiveness might be caused by 
point and deletion mutation of the siRNA target site ${ }^{[21,22]}$. An RNAi vector expressing multiple shRNAs is considered to be a valuable tool for highly effective gene suppression of single and multiple-genes targets, and can be used to prevent the viral escape by sequence mutation.

Pigs are important economic domestic animals, therefore breeding new lines that resistant to PEDV and TGEV would provide substantial benefits for pig producers. However, there have been no attempts to use RNAi-based technologies in pigs. In this paper, we developed a multiple shRNA technology that targets PEDV and TGEV, and produced transgenic pig embryos using somatic cell nuclear transfer (SCNT).

\section{Materials and methods}

\subsection{Sequence analysis of PEDV S gene in reference strains}

The full-length nucleotide sequences of PEDV spike (S) gene of 15 reference strains were characterized by sequence alignment and the conserved regions of the PEDV $\mathrm{S}$ gene were identified to enable shRNA design. The multiple sequence alignments and nucleotide sequence divergences were analyzed by DNAMAN 6.0 (Lynnon LLC, San Ramon, USA), which was also used to constructed an homology tree from the aligned nucleotide sequences.

\subsection{Construction of multiple shRNA expression vectors}

Two shRNAs were designed for PEDV. One shRNA, previously described by Wang et al. ${ }^{[18]}$, is located in PEDV ORF3. The other shRNA is located in the conserved region of the PEDV S gene. Two shRNAs for TGEV are described in Zhou et al. ${ }^{[19,20]}$. These shRNAs are designated as shRNA1, shRNA2, shRNA3 and shRNA4 (Table 1). The same single loop sequence was chosen for all the shRNAs. The basic structure is MluI-LoxP-hU6-shRNA1-shRNA2mU6-7SK-shRNA3-shRNA4-hH1-LoxP-SalI, which contains Eco31I + sense + loop + antisense + TTTTTT + $S a c I+E c o 31$ I, consisting of promoter, sense target, loop, complementary target and termination signal sequences in that order. The basic cassette was synthesized and inserted into the MluI/SalI sites of pGenesil-FUT1, as described in a previous study ${ }^{[23]}$, and the new construct was designated as pLoxP-TGEV-PEDV (Fig. 1).

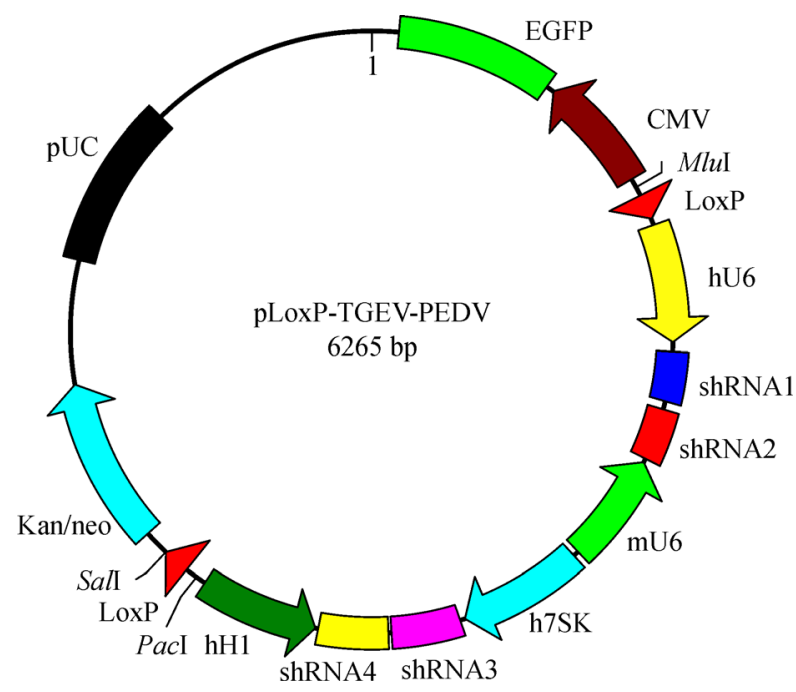

Fig. 1 Vector expressing four shRNAs targeting PEDV and TGEV driven by four different promoters (hU6, mU6, h7SK and $\mathrm{hH} 1)$

\subsection{Cell culture and production of transgenic cells}

Porcine fibroblast cells were isolated from 30 to $40 \mathrm{~d}$ old fetuses and were grown in culture medium (DMEM with $15 \%$ FBS) for two passages. Twenty-four hours before transfection, cells were seeded in each well of a six-well plate and cultured in growth medium without antibiotics to achieve greater than $80 \%-90 \%$ confluence. For transfection, the expression vector was digested by PacI and concentrated by NaAc, with $2 \mu \mathrm{g}$ of linear construct used per well. FuGENE ${ }^{\circledR} 6$ transfection reagent (Promega Corporation, Madison, WI, USA) was used according to the manufacturer's instructions. When the cells had reached confluence, the transfected cells were digested with $0.25 \%$ trypsin-EDTA, and the digested cells were

Table 1 shRNA sequences selected to design shRNA sequences for targeting PEDV and TGEV

\begin{tabular}{|c|c|c|}
\hline shRNA name & Oligo nucleotide sequence $\left(5^{\prime}-3^{\prime}\right)$ & Gene location \\
\hline shRNA1 & $\begin{array}{l}\text { 5'-CACCTGCAGTGATGTTTCTTGGATTCAAGACGTCCAAGAAACATCACTGCA TTTTTTG-3' } \\
\text { 5'-AGCTC AAAAAATGCAGTGATGTTTCTTGGA CGTCTTGAATCCAAGAAACATCACTGCA-3' }\end{array}$ & Spike \\
\hline shRNA 2 & $\begin{array}{l}\text { 5'-TTTGCCGAGTTGAGACATATATC TTCAAGACGGATATATGTCTCAACTCGG TTTTTTG-3' } \\
\text { 5'-AGCTC AAAAAACCGAGTTGAGACATATATCCGTCTTGAAGATATATGTCTCAACTCGG-3' }\end{array}$ & ORF3 \\
\hline shRNA 3 & $\begin{array}{l}\text { 5'-CCTCGTACTGGGATCGCACATATTTCAAGACGATATGTGCGATCCCAGTAC TTTTTTG-3' } \\
\text { 5'- AGCTCAAAAAAGTACTGGGATCGCACATATCGTCTTGAAATATGTGCGATCCCAGTAC-3' }\end{array}$ & Pol \\
\hline shRNA 4 & $\begin{array}{l}\text { 5'-TCCCGGCAAGAGCTCGTACAGTA TTCAAGACGTACTGTACGAGCTCTTGCC TTTTTTG-3' } \\
\text { 5'-AGCTC AAAAAAGGCAAGAGCTCGTACAGTA CGTCTTGAATACTGTACGAGCTCTTGCC-3' }\end{array}$ & Pol \\
\hline
\end{tabular}


transferred into $10-\mathrm{cm}$ plates. To produce transgenic cells, $800 \mu \mathrm{g} \cdot \mathrm{mL}^{-1} \mathrm{G} 418$ was added at $48 \mathrm{~h}$ to the medium for $16 \mathrm{~d}$, after which the complete fresh medium was added to the cells. The transgenic cell clones were allowed to reach confluence, following which they were collected for extraction of DNA to confirm shRNA integration, and then frozen in liquid nitrogen for somatic cell nuclear transfer.

\subsection{PCR of genomic DNA}

The transgenic cell DNA was extracted from the amplified clones, and the DNA was used as a PCR template. The following sequences were used as primers: 5'-GAGGGCCTATTTCCCATGAT-3' (sense); 5'-ATTTCCGGCTGTTTTGACAC-3' (antisense). These sequences are located in the promoter region and silencing target region of the shRNAs. The expected size of the target fragment was $778 \mathrm{bp}$. The PCR amplification conditions were $95^{\circ} \mathrm{C}$ for $4 \mathrm{~min}$, followed by 35 cycles of $94^{\circ} \mathrm{C}$ for $30 \mathrm{~s}, 55^{\circ} \mathrm{C}$ for $30 \mathrm{~s}$ and $72^{\circ} \mathrm{C}$ for $30 \mathrm{~s}$, followed by a final extension at $72^{\circ} \mathrm{C}$ for $7 \mathrm{~min}$. The PCR products were identified by agarose gel electrophoresis detection and then confirmed by sequencing.

\subsection{Production of transgenic SCNT porcine embryos}

For ovary collection and oocyte in vitro maturation (IVM), pig ovaries were collected from a local abattoir, and taken back to the laboratory in a thermos falsk containing saline at $37^{\circ} \mathrm{C}$. In the laboratory, ovarian follicles around 3-6 mm in diameter were aspirated using a $10 \mathrm{~mL}$ syringe connected to an 18 gauge needle. The aspirated follicular fluid was put into $15 \mathrm{~mL}$ centrifuge tubes to obtain cumulus-oocyte complexes (COCs). Only COCs with uniform ooplasm and investment of 2-3 layers of cumulus cells were selected for IVM in medium: TCM-199 supplemented with 5\% FBS, $10 \%$ porcine follicular fluid, $10 \mathrm{IU} \cdot \mathrm{mL}^{-1}$ of equine chorionic gonadotrophin, $5 \mathrm{IU} \cdot \mathrm{mL}^{-1}$ of human chorionic gonadotrophin, $0.8 \mathrm{mmol} \cdot \mathrm{L}^{-1}$ of L-glutamine, and $0.05 \mathrm{mg} \cdot \mathrm{mL}^{-1}$ of gentamicin, and incubated at $38.5^{\circ} \mathrm{C}$ and $5 \% \mathrm{CO}_{2}$ in air of maximum humidity. After IMV for $42 \mathrm{~h}$, the COCs were treated with $1 \mathrm{mg} \cdot \mathrm{mL}^{-1}$ hyaluronidase to denude the invested cumulus cells. Oocytes with clear perivitelline spaces, intact cell membranes, and extruded first polar body were selected for nuclear transfer.

For donor cells preparation, the frozen transgenic cells were thawed and washed in T2 (TCM-199 supplemented with $2 \%$ FBS), and only those cells showing round morphology and smooth surface and strongly refractivity under the microscope were used for nuclear transfer.

For the construction of somatic cell nuclear transfer embryos, nuclear donor transgenic cells and matured oocytes were placed in a drop of micromanipulation solution (T2 plus 5\% cytochalsin B), incubated at $100 \%$
RH for 10-15 min. Subsequently, one matured oocyte was fixed with a holding pipette, and the first polar body was removed with an injection pipette. Then, a transgenic somatic cell was injected into the perivitelline space through the same slot. For fusion and activation, the reconstructed couplets, after recovering for $30 \mathrm{~min}$ in $\mathrm{T} 2$, were transferred in batches to a fusion chamber and activated by two direct current pulses of $1.65 \mathrm{kV} \cdot \mathrm{cm}^{-1}$ with a 1-s interval. Finally after electrical activation, the couplets were washed in embryo culture media, transferred to chemically assisted activation medium: Porcine zygote medium 3 (PZM-3) with $10 \mu \mathrm{g} \cdot \mathrm{mL}^{-1}$ cycloheximide and $10 \mu \mathrm{g} \cdot \mathrm{mL}^{-1}$ cytochalasin B and incubated for $4 \mathrm{~h}$. Fusion was determined by examination under a stereomicroscope.

\subsection{PCR of embryos genomic DNA}

To confirm that the target shRNAs were inserted into the genome of the transgenic blastocysts, the blastocysts were boiled in water for $10 \mathrm{~min}$, then the products were used as a PCR template for amplification. The primers were 5'-GAGGGCCTATTTCCCATGAT-3' (sense) and 5'-ACTGCAGGTGTTTCGTCCTT-3' (antisense). The expected size of the target fragment of the vector was $255 \mathrm{bp}$. The PCR amplification conditions were $95^{\circ} \mathrm{C}$ for $4 \mathrm{~min}$, followed by 45 cycles of $94^{\circ} \mathrm{C}$ for $30 \mathrm{~s}, 55^{\circ} \mathrm{C}$ for $30 \mathrm{~s}$ and $72^{\circ} \mathrm{C}$ for $30 \mathrm{~s}$, followed by a final extension at $72^{\circ} \mathrm{C}$ for $7 \mathrm{~min}$. The PCR products were identified by agarose gel electrophoresis, and sequenced to confirm the target fragments were inserted into the genome of the blastocysts.

\section{Results}

\subsection{S gene nucleotide sequence analysis and homology tree}

The full-length nucleotide sequences of PEDV S genes of 15 field strains were determined to investigate their genetic diversity (Fig. 2). The sequences showed that the S genes of the 16 strains had 4146-4170 nucleotides (Table 2). These differences were related to the presence or absence of a number amino acids in the $\mathrm{N}$ terminus of different $\mathrm{S}$ proteins $^{[24]}$.

\subsection{Generation of transgenic porcine fibroblast cells}

After sixteen days selection, transgenic porcine fibroblasts cells were obtained and fresh complete medium was added to the cells. The transgenic cell clones were allowed to reach confluence, following which they were collected to extract DNA to confirm shRNA integration and then frozen in liquid nitrogen for somatic cell nuclear transfer. Most of the cells aged, lost their ability to grow, became grossly distorted and died after G418 selection. However, some colonies grew well and were transferred to $60-\mathrm{mm}$ 


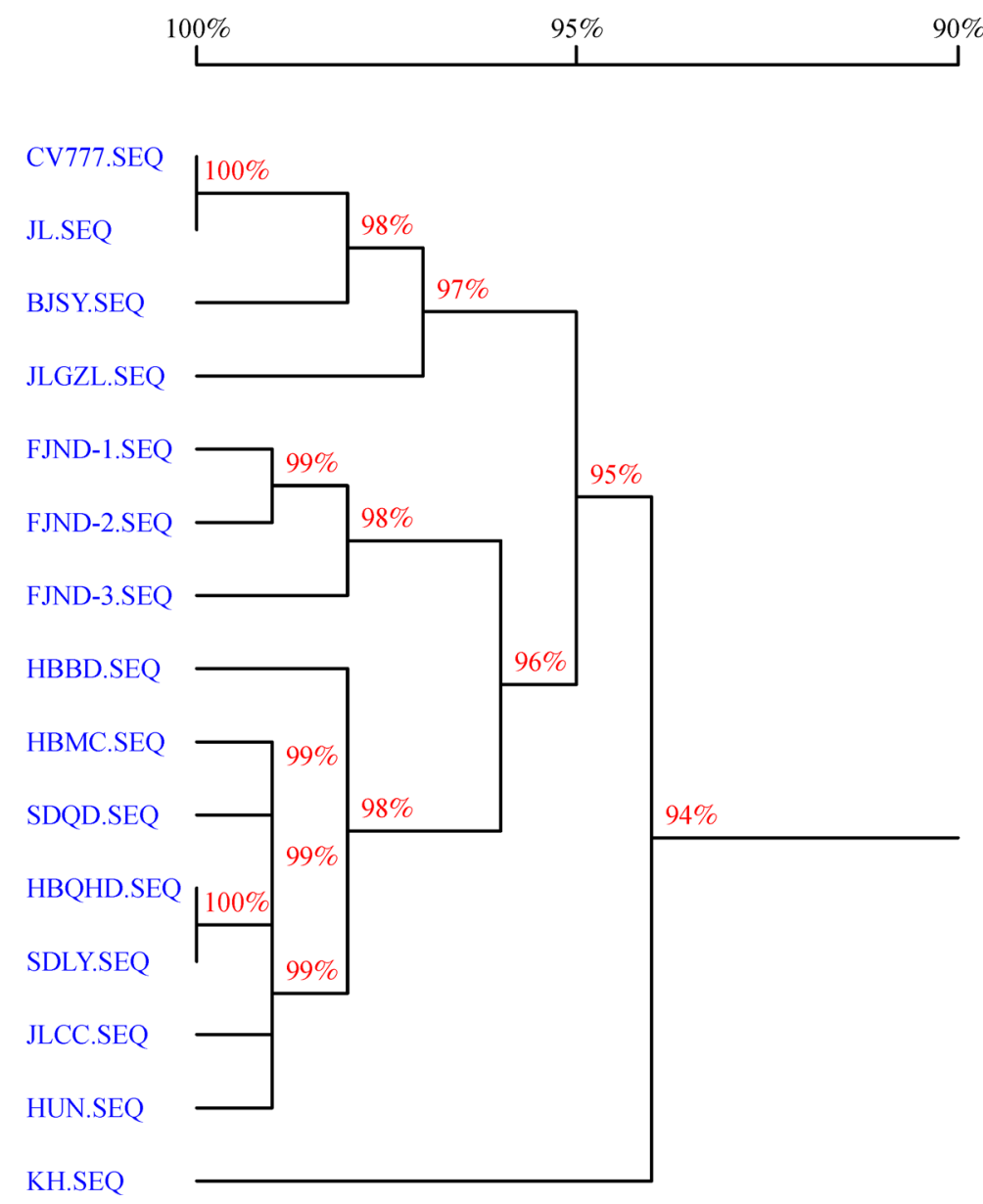

Fig. 2 S gene of 15 PEDV strains homology tree analysis

Table 2 S gene of 15 PEDV strains analysis

\begin{tabular}{lcccc}
\hline PEDV Field strains & Abbreviations & S gene/nt & S protein (aa) & Accession number \\
\hline CH-BJSY-2011-S & BJSY & 4149 & 1382 & JQ638921.1 \\
CH-FJND-1-2011-S & FJND-1 & 4149 & 1382 & JN543367 \\
CH-FJND-2-2011-S & FJND-2 & 4146 & 1381 & JN315706.1 \\
CH-FJND-3-2011-S & FJND-3 & 4161 & 1386 & JQ282909.1 \\
CH-HBBD-2011-S & HBBD & 4170 & 1389 & JQ638918 \\
CH-HBQHD-2011-S & HBQHD & 4161 & 1386 & JQ638922 \\
HBMC-2012-S & HBMC & 4161 & 1386 & JX163294.1 \\
CH-JL-2011-S & JL & 4149 & 1382 & JQ638924 \\
CH-JLCC-2011-S & JLCC & 4161 & 1386 & JQ638920 \\
CH-JLGZL-2011-S & JLGZL & 4152 & 1383 & JQ638923 \\
CH-SDLY-2011-S & SDLY & 4161 & 1386 & JQ638917 \\
CH-SDQD-2011-S & SDQD & 4161 & 1386 & JQ638919 \\
HuN-S & HuN & 4161 & 1386 & JQ517274.1 \\
CV777-S & CV777 & 4149 & 1382 & JN599150.1 \\
KH-S & KH & 4164 & 1387 & AB548622.1 \\
\hline
\end{tabular}


dishes to facilitate expansion. Expression of the enhanced reporter gene (EGFP) was checked by fluorescence (Fig. 3). Next, we detected the transgenic cells to further confirm the gene of interest. The results showed that the size of the target fragment was 778 bp (Fig. 4), confirming that the shRNAs had stably integrated into the genome of the transgenic porcine cells. Therefore, we inferred that these transgenic cells could be used for somatic cell nuclear transfer.

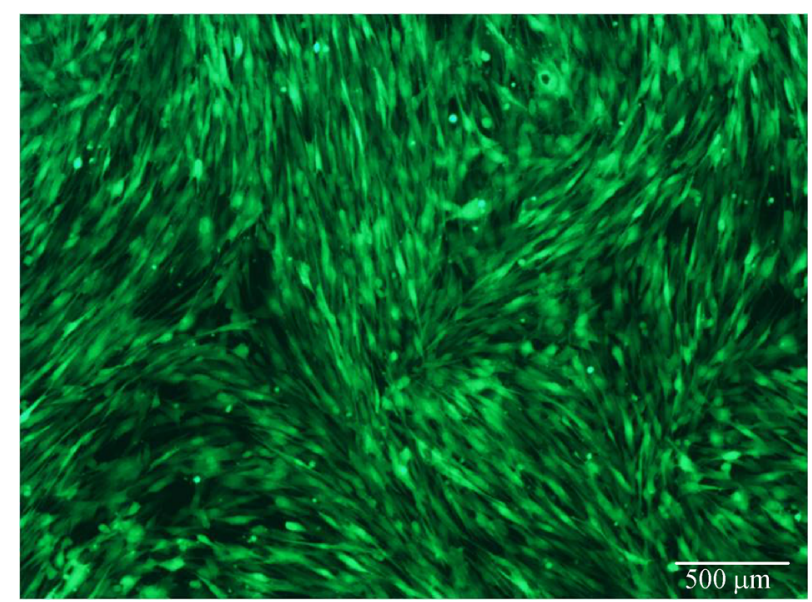

Fig. 3 The transgenic porcine fibroblast clones

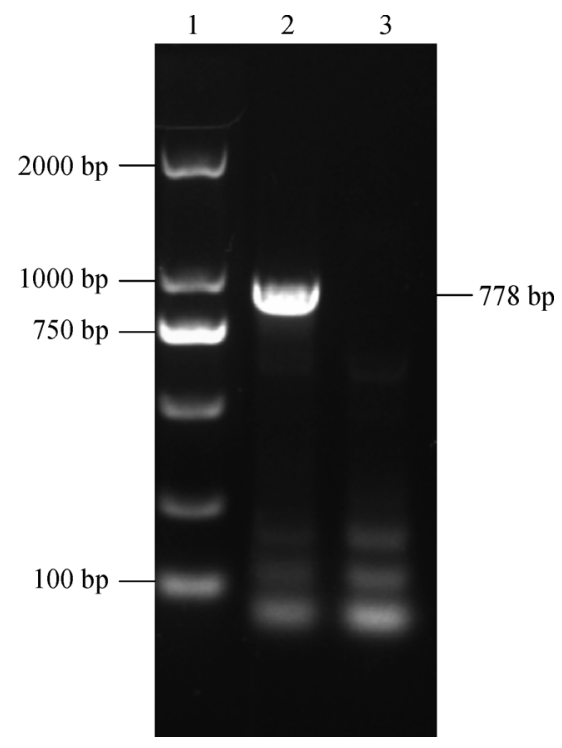

Fig. 4 Identification of transgenic porcine fibroblast clones by PCR. Lane 1, marker; Lane 2, transgenic porcine fibroblast; Lane 3 , non-transgenic porcine fibroblast.

\subsection{Development of SCNT embryos in vitro}

As shown in Fig. 5 , after 6 days the transgenic zygotes that expressed the EGFP had developed into blastocysts. Next, we tested the transgenic blastocysts to confirm the gene of interest was present. The result showed that the size of the

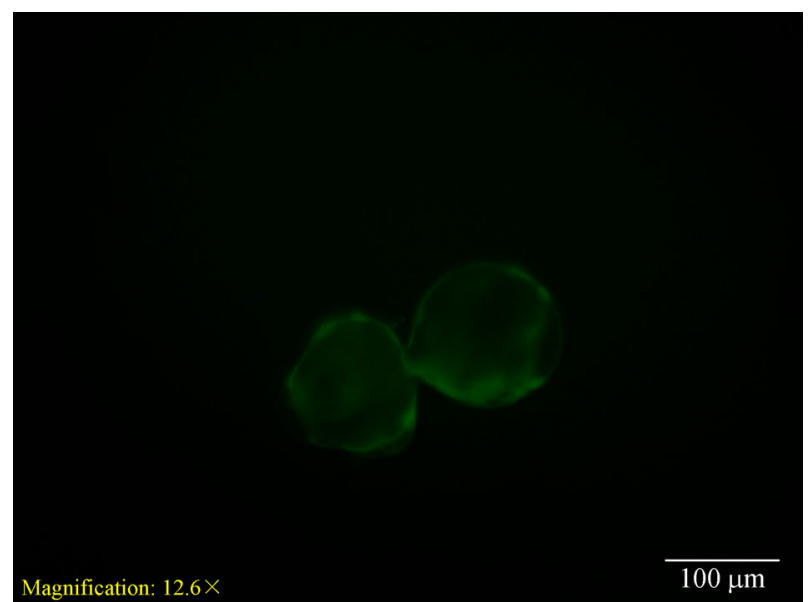

Fig. 5 Transgenic cloned embryos can be seen under a fluorescence microscope

target fragment was $255 \mathrm{bp}$, indicating that the shRNAs had stably integrated into the genome of the porcine blastocysts (Fig. 6) and this was confirmed by the PCR product sequencing (data not shown).

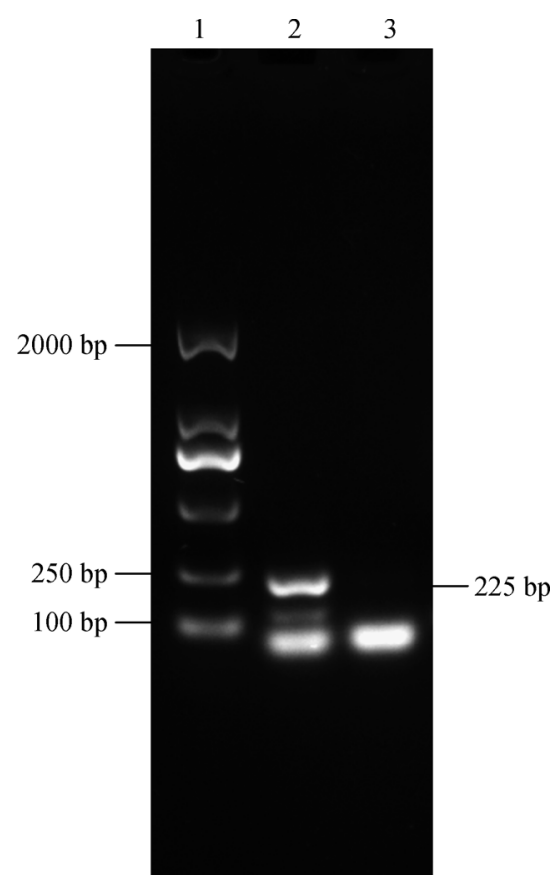

Fig. 6 Identification of transgenic porcine blastocyst by PCR. Lane 1, marker; lane 2, transgenic porcine embryo; lane 3, nontransgenic porcine embryo.

\section{Discussion}

For the development of an effective RNAi-based gene silencing system against PEDV and TGEV, a simple strategy would be to express multiple shRNA from multiple promoters in one vector ${ }^{[25,26]}$. There are four 
reasons for this. (1) Expression of multiple shRNAs inhibits gene expression and virus replication much more effectively than a single shRNA ${ }^{[26,27]}$. (2) Given the transfection efficiency, one vector more effectively expresses multiple shRNAs than can be achieved by cotransfection with multiple shRNAs vectors. (3) Multiple shRNAs can prevent viral escape because that would require acquisition of mutations in all shRNA-target sites $^{[21,22,28]}$. (4) Previous studies involving the expression of multiple shRNAs employing an expression strategy in which three or four shRNAs were expressed from the same promoters found that this caused frequent recombination at repeat sequences of the expression cassette, resulting in deletion of one or two of the cassettes ${ }^{[2]}$. To prevent such recombination, we selected four different promoters (hU6, mU6, h7SK and hH1) that lack sequence similarity and have well defined transcription start and termination sites for a multiple shRNAs expression strategy.

Pol and $\mathrm{N}$ genes in different virus strains are highly conserved, but the $\mathrm{S}$ gene is considered as the most useful for revealing the genetic diversity of coronavirus isolates $^{[29,30]}$. The PEDV S protein plays a critical role by interacting with the cellular receptor to mediate viral entry and inducing neutralizing antibodies in the natural host ${ }^{[31,32]}$. However, mutations resulting in viral escape are a big problem because shRNA loses its ability to inhibit virus replication ${ }^{[21,22]}$. Based on this principle, shRNA encoding multiple sequence variants that target sequences in a conserved region of the viral genome could usefully inhibit viral escape. In this study, shRNA1 previously described in Wang et al. ${ }^{[18]}$, is located in PEDV ORF3. The virus genome copies were reduced 4-fold compared to the control. The shRNA2 targeted the $\mathrm{S}$ genes of 15 PEDV strains. Two shRNAs designed to target TGEV, as described in previous studies, reduced the pre-transfected amounts of viral RNAs in cell cultures by $95.2 \%$ $100 \%{ }^{[19,20]}$. All the shRNAs were inserted into conserved regions of the main PEDV and TGEV strains to inhibit viral escape.

SCNT is one of the most effective strategies for transgenic production of large animals. In addition, this approach is very useful for studying issues associated with reproduction. The efficiency of transgene integration is extremely low, however, and selectable marker genes (SMG) are highly effective for increasing the transgenic cell identification rate. However, because SMGs are integrated into genome of transgenic animals, there are suggestions they may cause hazards to ecosystems and when products are consumed as food ${ }^{[33-37]}$, therefore the public is concerned about the biosafety of transgenic farm animals. It is imperative, therefore, to develop a technology that allows the production of transgenic farm animals without SMGs. The SMG-free technology has been universally applied in producing transgenic plants, but rarely in farm animals. In this study, to produce safe and healthy transgenic pigs, we have generated a modified a vector-based Cre-LoxP system, in which all SMGs (neomycin, kanamycin and EGFP) are flanked by LoxP sites. In other words, with use of Cre recombination, it will be possible to precisely eliminate all the SMGs from transgenic cloned pigs, which would shorten time needed to the evaluate biosafety and could be more readily accepted by consumers.

\section{Conclusions}

This is the first study targeting multiple sites in PEDV and TGEV simultaneously. We constructed a vector-based CreLoxP systems, containing four shRNAs which used four III promoters (hU6, mU6, h7SK and hH1), to generate transgenic SCNT porcine blastocysts. In the future, we will be generated transgenic pigs and assessed their resistance to PEDV and TGEV in vitro and in vivo.

Acknowledgements This work was supported by the National Transgenic Breeding Program of China (2014ZX08006-001B).

Compliance with ethics guidelines Jianwen Chen, Kaiyuan Pan, Zhen Chen, Biao Ding, Dandan Song, Wenbin Bao, and Yunhai Zhang declare that they have no conflicts of interest or financial conflicts to disclose.

All applicable institutional and national guidelines for the care and use of animals were followed.

\section{References}

1. Wood E N. An apparently new syndrome of porcine epidemic diarrhoea. Veterinary Record, 1977, 100(12): 243-244

2. Bi J, Zeng S, Xiao S, Chen H, Fang L. Complete genome sequence of porcine epidemic diarrhea virus strain AJ1102 isolated from a suckling piglet with acute diarrhea in China. Journal of Virology, 2012, 86(19): 10910-10911

3. Wang X M, Niu B B, Yan H, Gao D S, Yang X, Chen L, Chang H T, Zhao J, Wang C Q. Genetic properties of endemic Chinese porcine epidemic diarrhea virus strains isolated since 2010. Archives of Virology, 2013, 158(12): 2487-2494

4. Chen J, Liu X, Shi D, Shi H, Zhang X, Feng L, Song Y, Bi Y. Complete genome sequence of a porcine epidemic diarrhea virus variant. Journal of Virology, 2012, 86(6): 3408

5. Sun R, Cai R, Chen Y, Liang P, Chen D, Song C. Outbreak of porcine epidemic diarrhea in suckling piglets, China. Emerging Infectious Diseases, 2012, 18(1): 161-163

6. Chae C, Kim O, Choi C, Min K, Cho W S, Kim J, Tai J H. Prevalence of porcine epidemic diarrhoea virus and transmissible gastroenteritis virus infection in Korean pigs. Veterinary Record, 2000, 147(21): 606-608

7. Ren X, Glende J, Yin J, Schwegmann-Wessels C, Herrler G. Importance of cholesterol for infection of cells by transmissible gastroenteritis virus. Virus Research, 2008, 137(2): 220-224

8. Lyall J, Irvine R M, Sherman A, McKinley T J, Núñez A, Purdie A, Outtrim L, Brown I H, Rolleston-Smith G, Sang H, Tiley L. Suppression of avian influenza transmission in genetically modified 
chickens. Science, 2011, 331(6014): 223-226

9. Pfeifer A, Eigenbrod S, Al-Khadra S, Hofmann A, Mitteregger G, Moser M, Bertsch U, Kretzschmar H. Lentivector-mediated RNAi efficiently suppresses prion protein and prolongs survival of scrapieinfected mice. Journal of Clinical Investigation, 2006, 116(12): 3204-3210

10. Golding M C, Long C R, Carmell M A, Hannon G J, Westhusin M E. Suppression of prion protein in livestock by RNA interference. Proceedings of the National Academy of Sciences of the United States of America, 2006, 103(14): 5285-5290

11. Ramsoondar J, Vaught $\mathrm{T}$, Ball S, Mendicino M, Monahan J, Jobst $\mathrm{P}$, Vance A, Duncan J, Wells K, Ayares D. Production of transgenic pigs that express porcine endogenous retrovirus small interfering RNAs. Xenotransplantation, 2009, 16(3): 164-180

12. Chi H, Shinohara M, Yokomine T, Sato M, Takao S, Yoshida M, Miyoshi K. Successful suppression of endogenous $\alpha$-1,3-galactosyltransferase expression by RNA interference in pig embryos generated in vitro. Journal of Reproduction and Development, 2012, 58(1): 69-76

13. Bao Y, Guo Y, Zhang L, Zhao Z, Li N. Inhibition of porcine reproductive and respiratory syndrome virus replication by RNA interference in MARC-145 cells. Molecular Biology Reports, 2012, 39(3): 2515-2522

14. Luo J, Du J, Gao S, Zhang G, Sun J, Cong G, Shao J, Lin T, Chang H. Lentviral-mediated RNAi to inhibit target gene expression of the porcine integrin $\alpha \mathrm{v}$ subunit, the FMDV receptor, and against FMDV infection in PK-15 cells. Virology Journal, 2011, 8(1): 428

15. Tang D, Zhu H, Wu J, Chen H, Zhang Y, Zhao X, Chen X, Du W, Wang D, Lin X. Silencing myostatin gene by RNAi in sheep embryos. Journal of Biotechnology, 2012, 158(3): 69-74

16. Hu S, Ni W, Sai W, Zi H, Qiao J, Wang P, Sheng J, Chen C. Knockdown of myostatin expression by RNAi enhances muscle growth in transgenic sheep. PLoS One, 2013, 8(3): e58521

17. Hu S, Qiao J, Fu Q, Chen C, Ni W, Wujiafu S, Ma S, Zhang H, Sheng J, Wang P, Wang D, Huang J, Cao L, Ouyang H. Transgenic shRNA pigs reduce susceptibility to foot and mouth disease virus infection. eLife, 2015, 4: e06951

18. Wang K, Lu W, Chen J, Xie S, Shi H, Hsu H, Yu W, Xu K, Bian C, Fischer W B, Schwarz W, Feng L, Sun B. PEDV ORF3 encodes an ion channel protein and regulates virus production. FEBS Letters, 2012, 586(4): 384-391

19. Zhou J, Hua X, Cui L, Zhu J, Miao D, Zou Y, He X, Su W. Effective inhibition of porcine transmissible gastroenteritis virus replication in ST cells by shRNAs targeting RNA-dependent RNA polymerase gene. Antiviral Research, 2007, 74(1): 36-42

20. Zhou J, Huang F, Hua X, Cui L, Zhang W, Shen Y, Yan Y, Chen P, Ding D, Mou J, Chen Q, Lan D, Yang Z. Inhibition of porcine transmissible gastroenteritis virus (TGEV) replication in mini-pigs by shRNA. Virus Research, 2010, 149(1): 51-55

21. Westerhout E M, Ooms M, Vink M, Das A T, Berkhout B. HIV-1 can escape from RNA interference by evolving an alternative structure in its RNA genome. Nucleic Acids Research, 2005, 33(2): 796-804

22. Konishi M, Wu C H, Kaito M, Hayashi K, Watanabe S, Adachi Y, $\mathrm{Wu} \mathrm{G}$ Y. siRNA-resistance in treated $\mathrm{HCV}$ replicon cells is correlated with the development of specific HCV mutations. Journal of Viral Hepatitis, 2006, 13(11): 756-761

23. Chen J, Zhang Y, Zhang Y, Wei C, Liu X, Zhou N, Jia Q, Li Y, Zhang X, Zhang Y. Construction of multiple shRNAs expression vector that inhibits FUT1 gene expression and production of the transgenic SCNT embryos in vitro. Molecular Biology Reports, 2013, 40(3): 2243-2252

24. Chen J, Liu X, Shi D, Shi H, Zhang X, Li C, Chi Y, Feng L. Detection and molecular diversity of spike gene of porcine epidemic diarrhea virus in China. Viruses, 2013, 5(10): 2601-2613

25. ter Brake O, Konstantinova P, Ceylan M, Berkhout B. Silencing of HIV-1 with RNA interference: a multiple shRNA approach. Molecular Therapy, 2006, 14(6): 883-892

26. Brake O, Hooft K, Liu Y P, Centlivre M, Jasmijn von Eije K, Berkhout B. Lentiviral vector design for multiple shRNA expression and durable HIV-1 inhibition. Molecular Therapy, 2008, 16(3): 557-564

27. Ji J, Wernli M, Klimkait T, Erb P. Enhanced gene silencing by the application of multiple specific small interfering RNAs. FEBS Letters, 2003, 552(2-3): 247-252

28. Li J, Dai Y, Liu S, Guo H, Wang T, Ouyang H, Tu C. In vitro inhibition of CSFV replication by multiple siRNA expression. Antiviral Research, 2011, 91(2): 209-216

29. Penzes Z, Gonzalez J M, Calvo E, Izeta A, Smerdou C, Méndez A, Sanchez C M, Sola I, Almazan F, Enjuanes L. Complete genome sequence of transmissible gastroenteritis coronavirus PUR46-MAD clone and evolution of the purdue virus cluster. Virus Genes, 2001, 23(1): 105-118

30. Kim S J, Han J H, Kwon H M. Partial sequence of the spike glycoprotein gene of transmissible gastroenteritis viruses isolated in Korea. Veterinary Microbiology, 2003, 94(3): 195-206

31. Bosch B J, van der Zee R, de Haan C A, Rottier P J. The coronavirus spike protein is a class I virus fusion protein: structural and functional characterization of the fusion core complex. Journal of Virology, 2003, 77(16): 8801-8811

32. Chang S, Bae J, Kang T, Kim J, Chung G H, Lim C W, Laude H, Yang M, Jang Y S. Identification of the epitope region capable of inducing neutralizing antibodies against the porcine epidemic diarrhea virus. Molecules and Cells, 2002, 14(2): 295-299

33. Liu H, Jan M S, Chou C K, Chen P, Ke N. Is green fluorescent protein toxic to the living cells? Biochemical and Biophysical Research Communications, 1999, 260(3): 712-717

34. Krestel H E, Mihaljevic A L, Hoffman D A, Schneider A. Neuronal co-expression of EGFP and beta-galactosidase in mice causes neuropathology and premature death. Neurobiology of Disease, 2004, 17(2): 310-318

35. Huang W Y, Aramburu J, Douglas P S, Izumo S. Transgenic expression of green fluorescence protein can cause dilated cardiomyopathy. Nature Medicine, 2000, 6(5): 482-483

36. Daigle D M, McKay G A, Thompson P R, Wright G D. Aminoglycoside antibiotic phosphotransferases are also serine protein kinases. Chemistry \& Biology, 1999, 6(1): 11-18

37. Miki B, Abdeen A, Manabe Y, MacDonald P. Selectable marker genes and unintended changes to the plant transcriptome. Plant Biotechnology Journal, 2009, 7(3): 211-218 